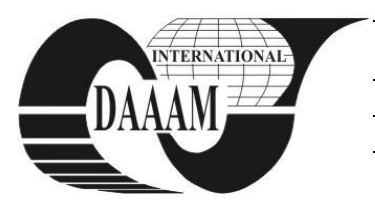

Annals of DAAAM for 2012 \& Proceedings of the 23rd International DAAAM Symposium, Volume 23, No.1, ISSN 2304-1382 ISBN 978-3-901509-91-9, CDROM version, Ed. B. Katalinic, Published by DAAAM International, Vienna, Austria, EU, 2012 Make Harmony between Technology and Nature, and Your Mind will Fly Free as a Bird Annals \& Proceedings of DAAAM International 2012

\title{
COMPARATIVE ANALYSIS OF DIFFERENT MECHANICAL STRUCTURES IN FUNCTIONAL OPTIMIZATION OF INDUSTRIAL ROBOTS NUMERICALLY CONTROLLED AXES
}

\author{
AVRAM, G[eorgia] - C[ezara]; NICOLESCU, A[drian] F[lorin]; \\ ENCIU, G[eorge] \& POPESCU, C[onstantin] - A[drian]
}

\begin{abstract}
Present work illustrates author's contributions on structural and functional analysis of numerical controlled (NC) axes of electric driving system industrial robots (IR). A complex assisted study on NC axes' optimum structure of a gantry robot for part handling application and both IR's $N C$ axes specific performance parameters and their influence on robot's overall performance has been performed. This article presents this study's $3^{\text {rd }}$ Stage. The research was aimed at identifying the optimal structure of an IRB's NC axis, this time modifying the mechanical structure and keeping the electrical structure proposed in the $2^{\text {nd }}$ Stage, in order to improve NC's axes / IR's performance level.

Keywords: Industrial Robots, numerically controlled axes, optimal design, electric driving system
\end{abstract}

\section{INTRODUCTION}

The results obtained in previous research work $\left(1^{\text {st }}\right.$ and $2^{\text {nd }}$ stages) for the structural and functional analysis of NC axes of electric driving system gantry industrial robots [1,2] and the results obtained for the 3rd stage (presented in this paper) are part of the study carried out so far for achieving the proposed objectives of the doctoral thesis titled "Research on structural and functional optimization of numerically controlled axes of the industrial robot, in order to increase their performances".

As specified in ISO 9283 [3] the overall IR's performance criteria evaluation should be carry out by selecting appropriate type and number of performance criteria in compliance with the specificity of robotic applications and the manufacturing. First mean to increase the IR's overall performance is related to structural and functional optimization of each IR's NC axes.

\section{SUBJECT FOR THE NC'S AXES STRUCTURAL AND FUNCTIONAL ANALYSIS}

The subject of analysis the authors propose is the three linear positioning axes of a GUDEL gantry robot for part handling application (Fig. 1).

The functional characteristics of the robot are as follows: Max Payload: 1000 [N]; Stroke on X axis: 5000 [mm]; Stroke on Y axis: 2000 [mm]; Stroke on Z axis: $1500[\mathrm{~mm}] ; X$ and $Y$ axis speed: $112.5[\mathrm{~m} / \mathrm{min}] ; Z$ axis speed: $67.5[\mathrm{~m} / \mathrm{min}]$; repeatability: $\leq 0.05[\mathrm{~mm}]$; drive system type electrical; an 1:6 gearbox transmission ratio for $\mathrm{X}$ and $\mathrm{Y}$ axis, and an 1:10 gearbox transmission ratio for $\mathrm{Z}$ axis. All axes have a rack-and-pinion intermediate mechanism for motion transformation.

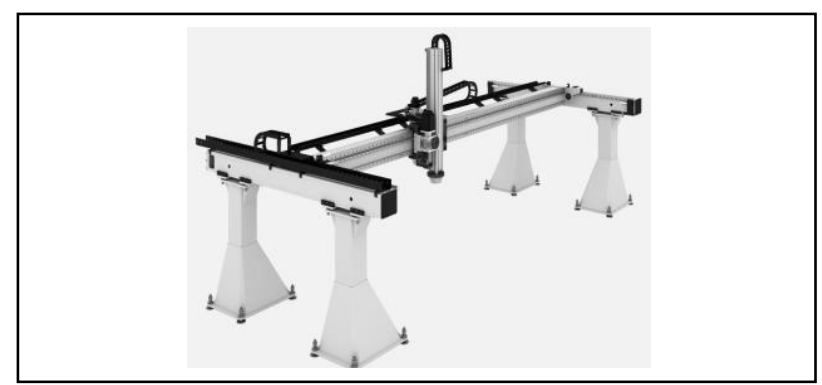

Fig. 1. GUDEL gantry robot - subject of the analysis

\section{CONSIDERED TERMS FOR THE NC'S AXES STRUCTURAL AND FUNCTIONAL ANALYSIS}

To define the optimal structure and the sizing of the IR's NC axis driving systems, the following elements should be taken into consideration [4]: Load; Orientation of the motion axis; Speed, acceleration and deceleration along with inertia forces applied to the moving element of the NC axis; Travel, Accuracy - in positioning and repeatability in the final index position; Environment; Duty Cycle - working cycle - motion profile - the ratio of operating and non-operating time and the influence of the static and dynamic behaviour of the IR's components and assemblies.

CAD steps for sizing full structure of the NC "Y axis" of the FP4 GUDEL gantry robot have been performed using "Motion Analyser ${ }^{\circledR}$ " (Rockwell Automation) software package. Below presented algorithm illustrates CAD steps flow - chart (Fig. 2).

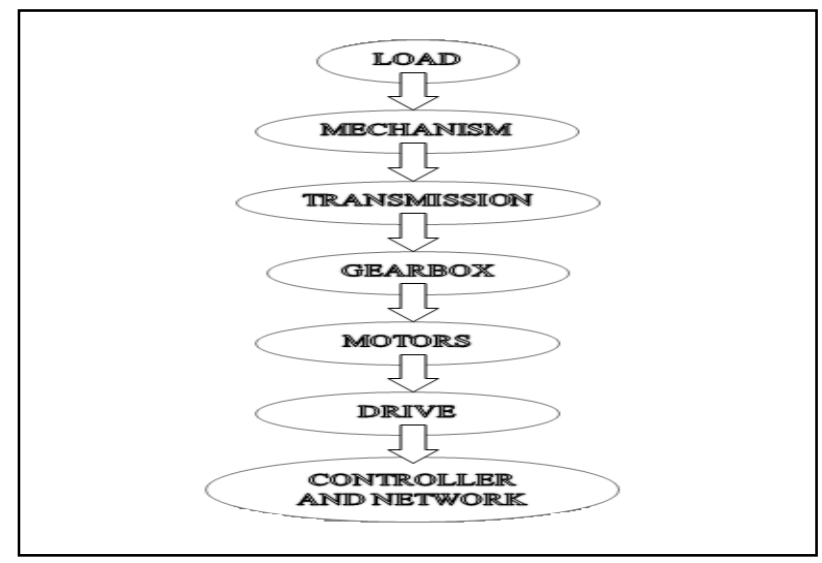

Fig. 2. CAD steps flow - chart 


\section{NC'S AXES STRUCTURAL AND FUNCTIONAL ANALYSIS}

For the final purpose of the NC's axes structural and functional analysis (to define and validate an assisted procedure for IR's NC axes optimal structure selection including both mechanical structure and electrical driving system - in accordance with IR's specific application and overall performance criteria) an assisted study on NC axes optimum driving motor's selection and IR's NC axes specific performance analyzes have been performed; the strategy used in this study is illustrated below:

\subsection{Stage 1. Analysis of IR's NC axes performance level for electromechanical structures of existing IR models.}

To preserve manufacturer's specifications for both the mechanical structure of the studied NC axis / robot model and the electrical driving system, the following steps have been completed [1], [2]:

Step 1.1: Define input data in compliance with the manufacturer's specifications for mechanical structure of studied NC axis and the electrical driving system;

Step 1.1.1: Introduce specific data into the simulation software for the mechanical structure of studied NC axis accordingly manufacturer's specifications for existing IR's model / design.

Step 1.2: Run simulation stage 1 (preserving manufacturer's specifications for the mechanical structure and electrical driving system of NC axes)

Step 1.2.1: Perform CAD specific procedure for selecting (in compliance with the manufacturer's specifications) the most appropriate electrical driving system available from the motors/servo drives data bases;

Step 1.2.2: Simulate electrical driving system's behaviour and overall $\mathrm{NC}$ axis' operation to evaluate NC's axes specific performance level;

Step 1.3: Results analysis

Step 1.3.1: Evaluate correspondence / differences between manufacturer's specifications for electrical driving system / NC's axes performance level versus simulation results;

Step 1.3.2: Conclusion on the NC's axes real performance level in case of preserving electrical driving system specified by manufacturer.

\subsection{Stage 2. Look for better solutions for the electrical driving system in reaching manufacturer's specifications for NC's axes performance level.}

After the $1^{\text {st }}$ stage has been completed, the analysis of IR's NC axes performance level continued with preserving the manufacturer's specifications for the mechanical structure of studied NC axes / robot model and look for improved electrical driving system solutions in order to increase NC's axes specific performance level [1]; [2].

Step 2.1: Run simulation stage 2 (preserving manufacturer specifications for the mechanical structure of NC axes)

Step 2.1.1: Evaluate, by means of simulation, the allowable alternatives for improved electrical driving system behaviour (considering existing NC's axes mechanical structure).
Step 2.1.2. Evaluate overall NC's axis performance level for new identified electrical driving systems and preserved NC's axes mechanical structure.

Step 2.2: Result's analysis.

Step 2.2.1: Conclude on overall simulation of the results obtained from the 2nd stage by recommending the manufacturer better alternatives for the electrical driving system selection in order to improve electrical driving system's behaviour and for achieving the increase of the overall NC's axis performance level (in terms of preserving the existing IR NC's axes mechanical structure).

\subsection{Stage 3. Analysis of IR's NC axes performance level where the modified mechanical structure and the electrical structure proposed in Stage 2 were considered.}

Going through steps similar to those registered for the $1^{\text {st }}$ and $2^{\text {nd }}$ Stages, (previously performed [1], [2]) the $3^{\text {rd }}$ Stage (actualy detailed) assumes identifying the IR's NC axes optimal structure by considering variation of both mechanical structure's parameters and electrical driving system behaviour, in order to further increase NC's axes specific performance level and recommend manufacturer certain means to further improve the IR's design.

During this stage, it was intended to replace the rackand-pinion type mechanism from the existing IR's NC axis structure analyzed in the $1^{\text {st }}$ and $2^{\text {nd }}$ stages, with a lead-screw type mecanism and make a comparative NC axis's behavior analysis, corresponding to each case.

Thus, in Table 1, the results obtained for the following two cases of $\mathrm{Y}$ NC's axis structure are depresented:

A.The mechanical structure of existing IR models [5] and the electrical structures proposed in the $2^{\text {nd }}$ Stage for the existing mechanical structure.

B. Electrical structures proposed in the $2^{\text {nd }}$ Stage and a mechanical structure with lead screw mechanism and no gearbox.

For case A, according to the robot manufacturer's specifications, the Pinion - Rack mechanism has been considered with the following specifications: $p=7.5$; module $=2.387 ; \mathrm{z}=20 ; \mathrm{L}=19.5 \mathrm{~mm} ; \mathrm{FN}=6.946 \mathrm{~N} ; \mathrm{TN}$ $=166 \mathrm{Nm}$; Pinion PCD: $47.746 \mathrm{~mm}$; Pinion Inertia: $0.38717 \mathrm{kgcm}^{2}$.

In both cases, the autors considered: Load: $49 \mathrm{~kg}$; Stroke: $2 \mathrm{~m}$; Velocity: $1.875 \mathrm{~m} / \mathrm{s}$, Additional Load Table/Slide Mass $=56 \mathrm{~kg}$, the motion profile presented in fig. 3, three motor families (MPL - Low Inertia Motor, MPM - Medium Inertia Motor, Elwood - Explosion Proof Servo Motor and the servo - drive ULTRA 3000 (compatible with previously selected motor family).

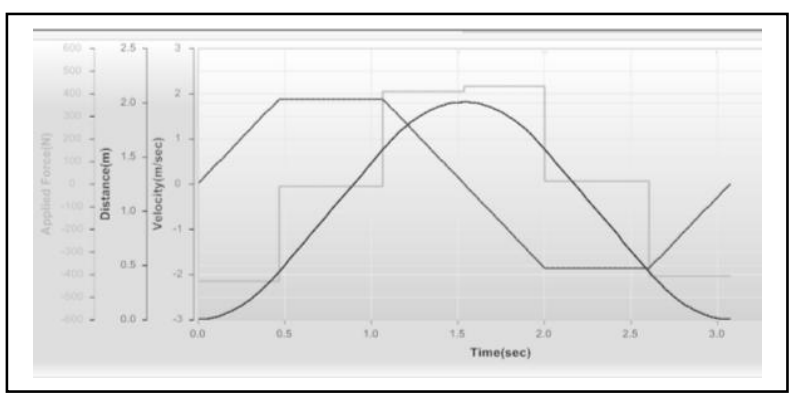

Fig. 3.The motion profile grafics 


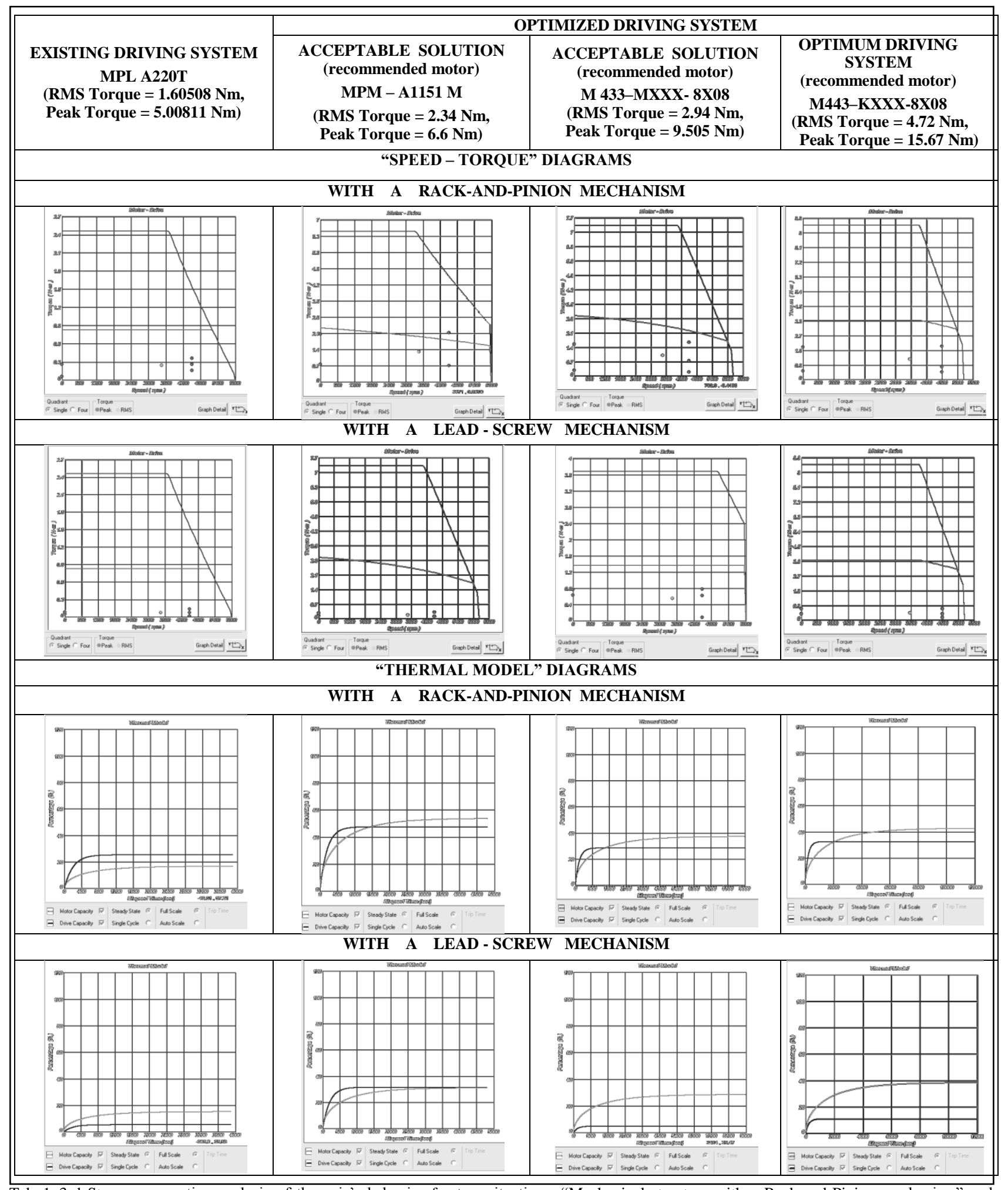

Tab. 1. 3rd Stage comparative analysis of the axis's behavior for two situations: "Mechanical structure with a Rack-and-Pinion mechanism" and "Mechanical structure with a Lead - Screw mechanism"

For case B, the transformation equation for the axis's mechanical structure was used to calculate the screw's lead $(25 \mathrm{~mm})$

$$
\begin{gathered}
\mathrm{Y}_{\mathrm{e}}=\mathrm{Y}_{\mathrm{i}} \times \mathrm{i}_{\mathrm{TLC}} \\
\mathrm{Y}_{\mathrm{e}}\left(\mathrm{V}_{\text {requob elem }}^{\max }\right)=\mathrm{Y}_{\mathrm{i}}\left(\mathrm{n}_{\mathrm{ME}}^{\max }\right) \times \mathrm{i}_{\text {GEARBox }} \times \mathrm{i}_{\mathrm{MT}} \\
\mathrm{i}_{1}=\mathrm{i}_{\mathrm{GEARBOX}}(=1: 6)
\end{gathered}
$$

$$
\mathrm{i}_{\mathrm{MT}}=\frac{\mathrm{m} \times \mathrm{z}_{\mathrm{p}}}{2}=\mathrm{R}_{\mathrm{p}}
$$

transmission ratio for rack-and-pinion mechanism and

$$
\mathrm{i}_{\mathrm{MT}}=\frac{\mathrm{p}}{2 \pi}
$$

transmission ratio for lead screw mechanism 


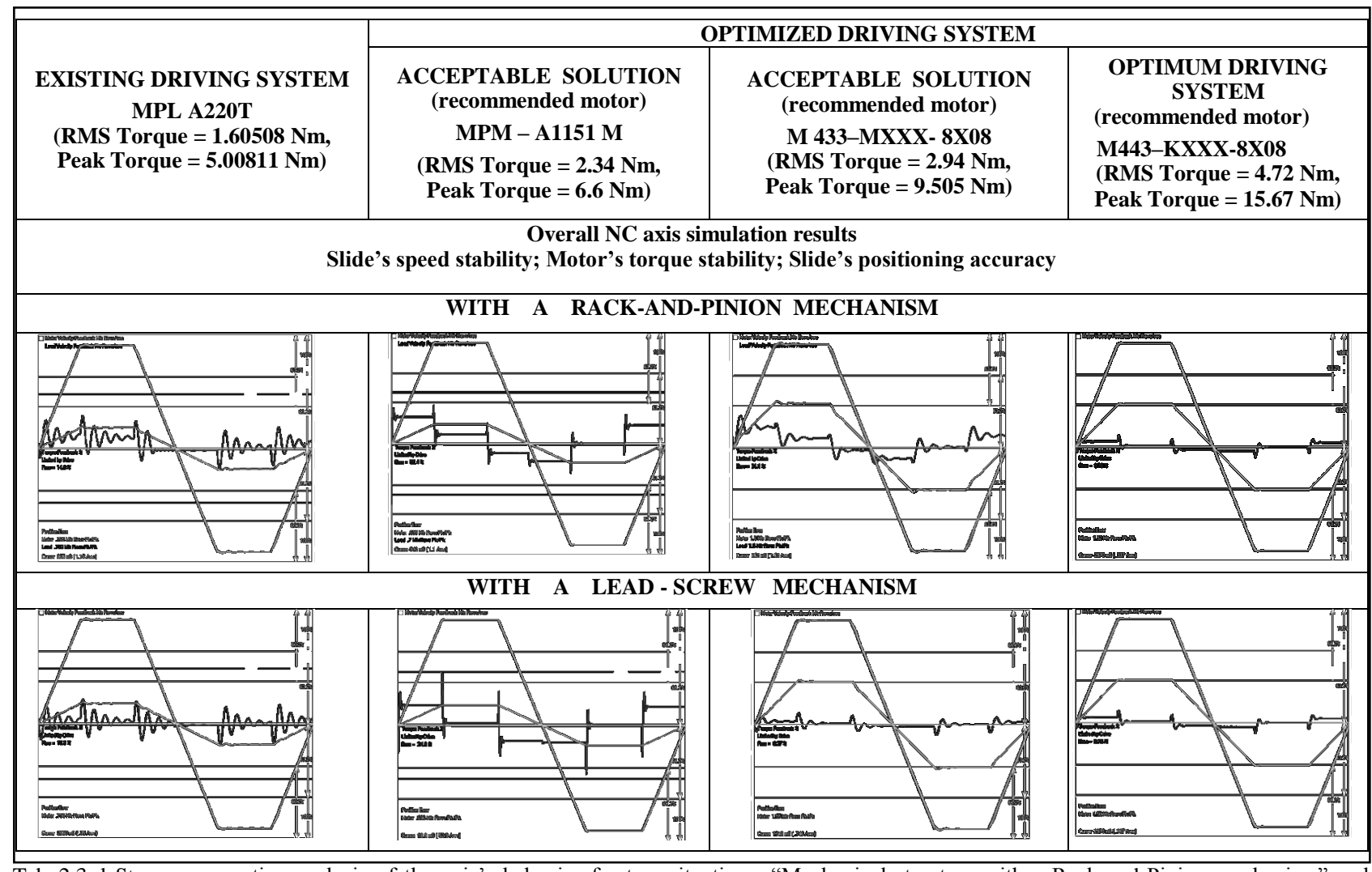

Tab. 2.3rd Stage comparative analysis of the axis's behavior for two situations: "Mechanical structure with a Rack-and-Pinion mechanism" and "Mechanical structure with a Lead - Screw mechanism"

\section{CONCLUSION}

Unitar criteria have been used for selecting the optimal solution during previously works $\left(1^{\text {st }}\right.$, the $2^{\text {nd }}$ stage) and actual works ( $3^{\text {rd }}$ stage) as follows: "Torque -Speed" Diagram (see Tab. 1), "Power - Speed" Diagram - the motor and servo - drive use, the motor thermal regime and servo-drive thermal regime; the "Simulation"'s results - an approximate simulation of the system's response during operation under operating conditions (see Tab. 1).

The research works developed in the $1^{\text {st }}$ and $2^{\text {nd }}$ Stage provides the manufacturer with solutions on how to improve NC's axes / IR's performance level in case of turning to better technical solutions identified for GUDEL FP 4 IR's electrical driving systems (already determined in terms of preserving the existing IR NC's axes mechanical structure). The present research works developed in the 3rd Stage provides information on the NC's axes / IR's performance level (see Tab. 1 - Overall $\mathrm{NC}$ axis simulation results) in case the rack-and-pinion mechanism and the gearbox were replaced using a leadscrew mechanism. Comparative analysis of different NC's axes performances level have been performed taking into account above mentioned two NC's axes structures.

Optimal solutions for NC Y axes of the GUDEL FP 4 robot - integrated in a part-handling application - were identified for 2 different situations: 1 - the optimal electrical driving system existing mechanical structure (corresponding to $1^{\text {st }}$ and $2^{\text {nd }}$ Stages) and 2 - the optimal electrical structure identified for the $1^{\text {st }}$ situation and the modified mechanical structure ( $3^{\text {rd }}$ Stage) which may be used to increase the individual performance of NC axis as well as the overall performance of the IR specific model performance. Further studies will be dedicated to generate a general optimizition algoritm and development of an associated expert-package software.

\section{ACKNOWLEDGEMENTS}

The work has been funded by the Sectoral Operational Programme Human Resources Development 2007-2013 of the Romanian Ministry of Labour, Family and Social Protection through the Financial Agreement POSDRU/107/1.5/S/76903.

\section{REFERENCES}

[1] Avram G.- C., A. Nicolescu, (2011). Contributions regarding structural and functional analysis of a gantry industrial robot nc axes, Proceedings in Manufacturing Systems, Vol. 6, ISSUE 3, November 10-11, Bucharest, ISSN 2067-9238, Ed Academiei Romane, pag 147-158, Bucharest

[2] Avram G.- C.; Nicolescu A. F.; Strajescu E. R. \& Petre M., (2011). Structural and Functional Optimization of Industrial Robot's NC Axes, "Annals of DAAAM for 2011 \& PROCEEDINGS of The 22nd DAAAM Symposium, Vol 22, No 1 - The 22nd DAAAM World Symposium "Intelligent Manufacturing \& Automation: Power of Knowledge and Creativity" and "The 5th European DAAAM International Young Researchers' and Scientists' Conference", 23-26 November 2011, AUSTRIA VIENA DAAAM International Vienna - Austria, Austria Center Vienna, Vienna, Austria, ISSN 1726-9679, ISBN 978-3-901509-83-4, pag. 0857-0858

[3] (1996) International Standard ISO 9283:1996, Manipulating Industrial Robots - Performance criteria and related test methods

[4] http://www.robotics.org/content-detail.cfm, (2009). Robots in Medical Applications: How LOSTPED and Cartesian Robots Can Help, Accessed: 2011-01-14

[5] http://www.gudel.com/modules/, GÜDEL Modules Catalog, D, F, E / 07.08 / NR. 0112130, (2008), Accesed: 2010-12-10 